\title{
Distribution of prostheses position after primary total knee arthroplasty on dominant and non- dominant sides
}

\section{ZHAO Fengchao}

the Affiliated Hospital ofZhejiang University https://orcid.org/0000-0002-7778-1779

LIU Lei ( $\sim 18326903127 @ 163 . c 0 m$ )

https://orcid.org/0000-0002-7076-8977

\section{Zha Guochun}

Affiliated Hospital of Xuzhou Medical University

Yang Guanjie

Affiliated Hospital of Xuzhou Medical University

\section{Xu Shizhuang}

Xuzhou Medical University

\section{Zheng Li}

Affiliated Hospital of Xuzhou Medical University

\section{Li Dadi}

Affiliated Hospital of Xuzhou Medical University

\section{Research article}

Keywords: Arthroplasty, replacement, knee, Prosthesis component positioning, Hand dominance

Posted Date: March 11th, 2020

DOI: https://doi.org/10.21203/rs.3.rs-16768/v1

License: (9) This work is licensed under a Creative Commons Attribution 4.0 International License.

Read Full License 
Distribution of prostheses position after primary total knee arthroplasty on dominant and non-dominant sides

Zhao Fengchao ${ }^{1}$, Liu Lei ${ }^{2 *}$, Zha Guochun ${ }^{2}$, Yang Guanjie ${ }^{2}, \mathrm{Xu}_{\text {Shizhuang }}{ }^{2}$, Zheng $\mathrm{Li}^{2}, \mathrm{Li} \mathrm{Dadi}^{2}$

(1. The First Affiliated Hospital of Zhejiang University, Hangzhou 310003, China

2. Department of Orthopaedics, the Affiliated Hospital of Xuzhou Medical University, Xuzhou 221002, China)

* Corresponding author: Liu Lei, E-mail: 18326903127@163.com

【Abstract】 Background To investigate the changes of prosthesis position after primary total knee arthroplasty (TKA) on the dominant and non-dominant sides. Methods A retrospective analysis was made of 132 patients (148 knees) who underwent primary TKA from December 2016 to December 2018 in our hospital. The patients were divided into dominant group (right side) and non-dominant group (left side) according to the position of the three right-handed surgeons. Total operation time, posterior distal femur angle (PDFA), posterior proximal tibia angle (PPTA), malposition rate of femoral prostheses in sagittal, postoperative hip-knee-ankle angle (HKA), mechanical lateral distal femoral angle (mLDFA), mechanical medial proximal tibial angle (mMPTA) were compared between the two groups. Results The mean total operation time was in dominant group was 111.8(111.8 \pm 26.9$)$ and in non-dominant group was $113.7(113.7 \pm 26.1)$ $(P>0.05)$. Postoperative sagittal alignment, the mean PDFA was $90.2\left(90.2^{\circ} \pm 3.3^{\circ}\right)$ in dominant group and $88.8\left(88.8^{\circ} \pm 3.6^{\circ}\right)$ in non-dominant group $(P<0.05)$. The mean PPTA was $84.8\left(84.8^{\circ} \pm 2.8^{\circ}\right)$ in dominant group and $85.4\left(85.4^{\circ} \pm 3.7^{\circ}\right)$ in non-dominant group $(P>0.05)$. The incidence of malposition of sagittal femoral prosthesis in dominant group was 6/76 (7.9\%) and that in non-dominant was $15 / 72(20.8 \%)$. There was a significant difference between the two groups $\left(\chi^{2}=5.083, P=0.024\right)$. Postoperative coronal alignment, the mean angle of components placement was $2.1^{\circ} \pm 4.2^{\circ}$ for HKA, $88.7^{\circ} \pm 2.7^{\circ}$ for mLDFA, $89.5^{\circ} \pm 2.7^{\circ}$ for mMPTA in dominant group and $1.6^{\circ} \pm 4.1^{\circ}$ for HKA, $89.0^{\circ} \pm 2.8^{\circ}$ for mLDFA, $89.9^{\circ} \pm 2.7^{\circ}$ for mMPTAin non-dominant group, respectively. There were no significant difference between the two groups ( $t=0.804$ $P=0.423, t=-0.564 P=0.574, t=-1.011 P=0.314$ ). Conclusion Our study suggests that surgeon handedness is likely to be one of factors that affect the sagittal placement of femoral component. Orthopaedic surgeons should be aw are of the potential problems and consider taking extra precautions to mitigate the risk before performing surgery.

【Key words 】 Arthroplasty, replacement, knee, Prosthesis component positioning, Hand dominance

\section{Background}

Total Knee Arthroplasty (TKA) is an effective method for the treatment of end-stage knee arthritis, and ensuring the accurate positioning and alignment of knee components has been considered associated with good stability and a lower rate of loosening ${ }^{1 ; 2}$. Most of studies focus on improving the accuracy of prosthesis placement including computer-assisted navigation systems, robotic-assisted systems, and patient-specific instrumentation during the past decade ${ }^{3}$.

But there are few studies about the influence of dominant side of the surgeon. Moloney et al. ${ }^{4}$ compared preoperative and postoperative radiographs of 244 patients with femoral neck or intertrochanteric fractures treated with sliding hip screws and found that 12 surgical failures occurred on the left. Pennington et al.'s ${ }^{5}$ study suggested that the surgeon's dominant hand had an influence on the position of the acetabular component. Another recent study has shown that right-handedness is a problem during the upper thoracic pedicle screw placement on the left side 
by retrospectively reviewing 33 patients ${ }^{6}$.

At present, it is still unknown whether the surgeon's dominant side and non-dominant side have influence on the position of the knee prosthesis. Even though computer-assisted navigation technology can obtain more accurate components alignment than conventional surgical techniques, there is no statistically significant difference in terms of clinical outcome ${ }^{7}$. Moreover, the navigation systems are more expensive and the operation times are longer than conventional techniques. Conventional TKAs are still the main method for the treatment of end-stage knee arthritis. Thus, the purpose of this study was to investigate whether surgeons dominant side had any influence in primary TKA components alignment based on postoperative radiography. We hypothesize that the surgeon's dominant side has an influence on components placement and it is more accurate in dominant side than non-dominant side.

\section{Methods}

We prospectively followed up and retrospectively reviewed 531 consecutive patients (531 knees) who accepted primary total knee arthroplasties from May 2016 to December 2018.(Fig.1).The TKAs were performed by 3 surgeons(A, B, and C), who were defined as right-handers according to the Edinbuegh Handedness Inventory ${ }^{8}$. The annual operation volume of surgeons were more than 50 TKAs/year ${ }^{9}$. All patients were placed in the supine position, the surgeons performed the surgical procedure standing on the side of the operative extremity. We defined the right-handed surgeons performed the right knee replacement as the dominant side, and the left knee replacement performed by right-handed surgeons was defined as the non-dominant side.

All operations were performed through the medial incision of the knee joint and medial paraptellar approach, the patellofemoral joint was exposed after turning outward the patella, The distal femoral was cut using intramedullary rods and the proximal tibial using extramedullary alignment guides. A posterior reference point of the femur was used as a guide to determine the position of the femoral component in the sagittal plane. Cemented, posterior-stabilized TKAs were performed after use of a measured resection technique. The operation time was recorded from incision to skin closure. All patients stayed until the surgical suture was removed.

Patient demographics, diagnosis, degree of deformity, surgeon, side, prosthesis type, total operation time, complications were recorded. Radiographic analysis was performed on full-length standing anteroposterior radiograph of the lower extremity and adequate lateral radiographs using a picture archiving and communication system (PACS) measurement tools on the earliest acceptable postoperative images. Radiographs were measured by two independent observers who had no clinical contact with the patients.

The following angles were used for descriptive analys is of pre- and postoperative alignment: The hip-knee-ankle angle (HKA) was defined as the angle between the femoral mechanical ax is and the mechanical axis of the tibia. The hip center was obtained using concentric Moose circles ${ }^{10}$. The pre-operative center of knee was measured by the intersection of the midline between the tibial spines and the midline between the femoral condyles and tip of the tibia. The center of the ankle was determined as the midpoint of the talus. Postoperatively, the center of the knee was determined as the intersection of the midline in the middle of the poly-ethylene inlay and the midline between the condyles of the femoral prosthesis and the tip of the tibial prosthesis ${ }^{11}$. The HKA was defined to be normal between $\pm 3^{\circ}$ of the neutral alignment ${ }^{12 ; 13}$. The mechanical lateral distal femoral angle (mLDFA) was defined as the lateral angle between the mechanical ax is 
of the femur and the distal femoral articulate surface. The mechanical medial tibial angle (mMPTA) was defined as the medial angle between the mechanical of tibia and the proximal tibial joint line (Fig.2.1). The ideal postoperative angle was $90^{\circ} 14$.

The posterior distal femur angle (PDFA) was defined as the angle between the femoral posterior diaphyseal cortical bone and the distal femoral cut, The posterior proximal tibia angle (PPTA) was defined as the angle between the tangent to the posterior diaphyseal cortical bone and the tibial plateau. They were measured using published methods ${ }^{14}$ (Fig.2.2). The normal angle was $90^{\circ}$. In addition, the femoral component that was placed unevenly with the distal part of the native femur was defined as malposition of femoral component including over-flexed femoral prosthesis and femoral notch.

Statistical analysis

All data were analyzed using SPSS version 19.0 (IBM). Interobserver reliabilities were assessed by the interclass correlation coefficient (ICC). Univariate analysis was performed using parametric (Student $t$ test) such as age, body mass index (BMI), HKA, mLDFA, mMPTA, PDFA, PPTA between dominant and non-dominant sides. Nominal categorical variables such as male were assessed using the Chi-square or Fisher exact test. A p value of $<0.05$ was considered statistically significant.

\section{Result}

A total of 132 patients (148 knees) were enrolled in this study. There were 25 males (27 knees) and 107 females (121 knees). The mean age was 67.3 years, BMI was $26.6 \mathrm{~kg} / \mathrm{m}^{2}, 72$ were left side and 76 were right side. The main diagnos is was osteonecrosis of the knee osteoarthritis (92.6\%, 137/148). The operations of surgeon A, B, and C were: 100 knees, 31 knees, and 17 knees, respectively. The majority type of implants involved were post-stabilized prostheses (Zimmer Nex Gen LPS) $(31.8 \%, 47 / 148)$ (Table 1). There were no statistically significant differences in preoperative age, gender, BMI, and HKA between the dominant and non-dominant groups (Table 2).

The interclass coefficients for independent measurement by two observers of limb and knee alignment were reliable. The interobserver reliability was 0.87 (95\% confidence interval $[\mathrm{CI}] 0.83$; $0.89)$ for preoperative HKA and $0.99(95 \%$ CI $0.9 ; 0.99)$ for postoperative HKA. The interobserver reliability was $0.98(95 \%$ CI $0.96 ; 0.98$ ) for mLDFA and $0.98(95 \%$ CI $0.95 ; 0.98$ ) for mMPTA. The interobserver reliability was $0.88(95 \%$ CI 0.83 ; 0.91$)$ for PDFA and $0.87(95 \%$ CI 0.83 ; 0.90$)$ for PPTA, respectively.

\section{Postoperative coronal alignment}

Analys is of HKA revealed a mean of 2.1 degrees on the dominant operated side and 1.6 degrees on the non-dominant side. Statistical analysis using Independent-Samples t-test showed this to be not significant $(P=0.423)$. The mean mMPTA was 89.5 degrees on the dominant side and 89.9 degrees on the non-dominant side. The mean mLDFA was 88.7 degrees on the dominant side and 89.0 degrees on the non-dominant side. Independent-Sample t-test also found this to be not statistically significant $(P=0.314, P=0.574$ respectively) in terms of the postoperative coronal alignment of the components.

\section{Postoperative sagittal alignment}

The mean PDFA represented postoperative sagittal alignment of femoral component was on the dominant operated side and on the non-dominant side. The mean PDFA was $90.2\left(90.2^{\circ} \pm 3.3^{\circ}\right)$ in dominant group and $88.8\left(88.8^{\circ} \pm 3.6^{\circ}\right)$ in non-dominant group. There was statistically difference 
in two groups $(P=0.015)$. We further analyzed the proportion of the ideal position of the femoral prosthesis in the sagittal plane between the two sides and found the odds ratio of the dominant side was $72.4 \%(55 / 76)$, and the non-dominant group was $69.4 \%(50 / 72)$. The difference was not statistically significant $\left(\chi^{2}=0.153, P=0.695\right)$ (Fig.3). The mean PPTA represented postoperative sagittal alignment of tibial component was on the dominant operated side and on the non-dominant side. The t-test showed this to be not significant $(P=0.278)$ (Table 3).

The incidence of femoral notch was $1.3 \%$ in the dominant operated side and $9.7 \%$ in the non-dominant side. This difference approached but did not reach statistical significance $\left(\chi^{2}=3.598\right.$, $P=0.058$ ). The incidence of over-flexed femoral prosthesis in the dominant side was $6.5 \%$, and that in the non-dominant side was $11.1 \%$. There was no significant difference between the two groups $\left(\chi^{2}=0.948, P=0.330\right)$. But the rate of malposition of femoral prosthesis was $7.9 \%$ in dominant side compared with $20.8 \%$ in non-dominant side. The difference between the two groups was statistically significant $\left(\chi^{2}=5.083, P=0.024\right)$. There was statistically significant difference between the sides for malposition of femoral component.

\section{Total operative time between the sides}

The total operative time of the dominant side was (111.8 \pm 26.9$)$ min, and the non-dominant side was $(113.7 \pm 26.1) \mathrm{min}$. The mean operative time of TKA in the dominant side was shorter than that of the non-dominant side, but the difference between the sides was not statistically significant. ( $t=-0.422, P=0.674)$.

\section{Discussion}

Our study suggests that there was a significant difference on the sagittal alignment of femoral prosthesis between dominant and non-dominant side TKAs performed by right-handed surgeons. Several studies about the influence of surgeon's handedness have been reported in total hip arthroplasty (THA). Song et al. ${ }^{15}$ evaluated the placement of postoperative acetabular prosthesis and found that the right-handed surgeons put the acetabular cup more ideal and accurate in right THA than in left THA. They hypothesized that it is more comfortable and convenient for a right-handed surgeon to operate right THA than on the opposite side. Similarly, when the right-handed surgeons performed TKAs, they also faced the problem of the dominant and the non-dominant sides. At present, there are few studies on the relationship between the surgeon's dominant side and the position of the prostheses after primary total knee arthroplasty.

In Mehta et al.'s ${ }^{16}$ study, they found that there were statistical differences in extension and KS Function and Pain scores in patients who underwent TKAs performed by a right-handed surgeon standing on the side of the operative extremity. They thought this may be related to dexterity or proprioception and should considerate laterality of operative site with respect to surgeon handedness as a factor affecting task performance and outcome. Our study suggested that the malposition rate of sagittal femoral prosthesis in non-dominant side was significantly higher than dominant side. Sagittal plane positioning and alignment of the femoral component were associated with anterior knee pain according to Scott CEH et al's study ${ }^{17}$. However, Hsieh et al. ${ }^{18}$ retrospectively analyzed 233 patients accepting primary total knee arthroplasty and found that there was no statistical difference in the bony procedures of tibial resection and femoral cutting between left and right TKA performed by right-handed surgeons. In addition, the thicknesses of the polyethylene were not significantly difference between two groups. They concluded that the right-handed orthopedic surgeons successfully performed TKA procedures to achieve bone resection, soft-tissue balance and thicknesses of the polyethylene between left and right extremity. 
But we studied the placement angles of component by comparing the postoperative coronal and sagittal alignment of knee prosthesis in left and right side and might better reflect the operation of surgeons in TKA.

At present, the reasons for the different effects of right-handed surgeons performed on left and right side are not clear. But we have posed a possible hypothesis. In our study, all patients who accepted primary TKA were placed in supine position. During right TKA, the right-handed surgeons often choose entry point by holding the drill with their right hands. However, the entry point was chosen by right-handed surgeons with their left hand in left TKA for convenience. In these cases, the positions of the entry point in left TKA are higher or lower than right TKA. This difference of entry points in left and right TKAs may result in malposition of femoral prosthesis in sagittal. In addition, we found no significant differences of total operative time between the two sides.

This study has several limitations: (1) This is a retrospective and single-center study, which may have uncontrolled confounding factors which can result in marked bias. (2) All parameters were measured on X-ray films and no measurement of implant rotation. (3) Three surgeons included in our study were right-handers, which probably results in one-sidedness of the result. Further research would be conducted to eliminate the above limitations.

\section{Conclusion}

The results of this study suggest that surgeon handedness is likely to be one of factors that affects the placement of femoral component, especially with regard to the postoperative sagittal alignment of the femoral component. The placement of femoral component performed by the dominant hands of surgeons is more accurate than that performed by the non-dominant sides. The rate of malposition of femoral prostheses in the non-dominant side is higher than that in the dominant side. Orthopaedic surgeons should be aware of the potential problems that may be introduced by their handedness and the laterality of operated extremities and consider taking extra precautions to mitigate the risk before performing surgery.

\section{Abbreviations}

TKA: total knee arthroplasty; HKA: hip-knee-ankle angle; PDFA: posterior distal femur angle, PPTA: posterior proximal tibia angle; mLDFA: mechanical lateral distal femoral angle; mMPTA: mechanical medial proximal tibial angle; PACS: picture archiving and communication system; BMI: body mass index; ICC: interclass correlation coefficient; THA: total hip arthroplasty.

\section{Ack nowledgements}

The authors thank Qi Cheng, Xin Zheng, Yong Pang, Yeshuai Shen, Jinlong Tang, from the Department of Orthopedic Surgery, Affiliated Hospital of Xuzhou Medical University for their technical support.

\section{Authors' contributions}

FCZ performed the study, analyzed the data, and wrote the manuscript. GJY and SZX helped in the revising of the manuscript. LZ, GCZ and DDL helped in the collecting of the data. LL was involved in the study design, and made meaningful suggestions. All authors reviewed and approved the final submitted version.

\section{Funding}

This work was supported by the National Natural Science Foundation of China(81672184).

\section{Availability of data and materials}

All data generated or analyzed during this study are included in this published article. We do not 
wish to share our patients' data because it involves patient's privacy.

\section{Ethics approval and consent to participate}

This study has been approved by the Ethic Committee of the Affiliated Hospital of Xuzhou Medical University (NO.XYFY2019-KL111-02). Informed consent was obtained from all patients included in the study.

\section{Consent for publication}

We obtained permission from patients to publish their data, and all authors consent for publication.

\section{Competing interests}

The authors declare that they have no competing interests.

\section{Reference}

1. Fang DM, Ritter MA, Davis KE. Coronal alignment in total knee arthroplasty: just how important is it? Journal of Arthroplasty 24:39-43.

2. Sikorski JM. 1995. Alignment in total knee replacement. Instructional Course Lectures 44:455 .

3. Siddiqi A, Hardaker WM, Eachempati KK, et al.2017. Advances in Computer-Aided Technology for Total Knee Arthroplasty. Orthopedics:1.

4. Moloney D, Bishay M, Ivory J, et al. 1994. Failure of the sliding hip screw in the treatment of femoral neck fractures: 'Left-handed surgeons for left-sided hips'. Injury-international Journal of the Care of the Injured 25 Suppl 2:B9.

5. Pennington N, ., Redmond A, ., Stewart T, ., et al. 2014. The impact of surgeon handedness in total hip replacement. Annals of the Royal College of Surgeons of England 96:437.

6. Yaman O, Acaroğlu E. 2014. Role of surgeon handedness in transpedicularscrew insertion. Acta Orthop Traumatol Turc 48:479-482.

7. Roberts TD, Clatworthy MG, Frampton CM, et al.2015. Does Computer Assisted Na vigation Improve Functional Outcomes and Implant Survivability after Total Knee Arthroplasty? Journal of Arthroplasty 30:59-63.

8. RC O. 1971. The assessment and analysis of handedness: the Edinburgh inventory. Neuropsychologia 9:97-113.

9. MalikA, Mufarrih S, Ali A. 2017. Does surgeon volume affect the outcome of total knee arthroplasties in a developing country? - A retrospective cohort study. Annals of Medicine and Surgery:38-43.

10. Cooke TDV, Sled EA, Scudamore RA. 2007. Frontal plane knee alignment: A call for standardized measurement. The Journal of Rheumatology 34:1796-1801.

11. Vanlommel L, Vanlommel J, Claes... S. Slight undercorrection following total knee arthroplasty results in superior clinical outcomes in varus knees. 21:2325-2330.

12. ParratteS, Pagnano MW, Trousdale RT, et al.2010. Effect of postoperative mechanical axis alignment on the fifteen-year survival of modern, cemented total knee replacements. J Bone Joint Surg Am 92:2143-2149.

13. RITTER MA, FARIS PM, KEATING EM, et al.1994. Pos toperativeAlignment of Total Knee Replacement Its Effect on Survival. Clinical Orthopaedics and Related Research 299:153-156.

14. Heyse TJ, Decking R, Davis J, et al. Varus Gonarthrosis Predisposes to Varus Malalignment in TKA. 5:143-148.

15. Song X, Ni M, Li H, et al. 2018. Is the cup orientation different in bilateral total hip arthroplasty with right-handed surgeons using posterolateral a pproach? Journal of 
Orthopa edic Surgery and Research 13.

16. Samir M, Lotke PA. 2007. Impact of surgeon handedness a nd laterality on outcomes of total knee arthroplasties: should right-handed surgeons do only right TKAs? Am J Orthop 36:530-533.

17. Scott CEH, Clement ND, Yapp LZ, et al. 2019. Association Between Femoral Component Sagittal Positioning and Anterior Knee Pain in Total Knee Arthroplasty. The Journal of Bone and Joint Surgery 101:1575-1585.

18. Hsieh J, Lan M, Chang Y, et al. 2018. The Association between Surgery Laterality in Orthopedists and the Choices of Prostheses in Total Knee and Hip Replacement. Open Journal of Orthopedics 08:257-266.

\section{Appendix}

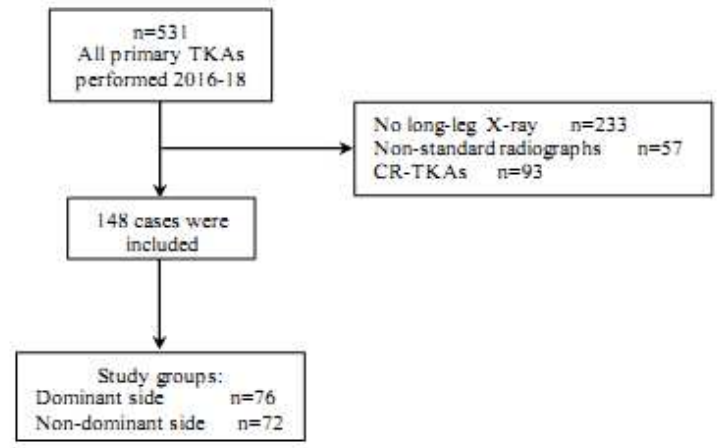

Fiğ I Study details TKA=total knee arthroplasty, CR=cruciate-retaining

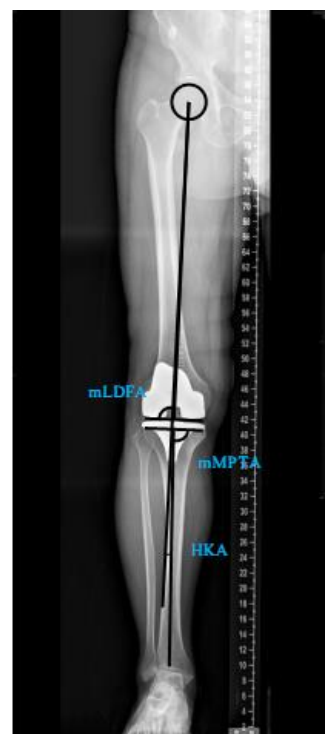

Fig2.1 Method for measuring HKA,mLDFA and mMPTA on the full radiography

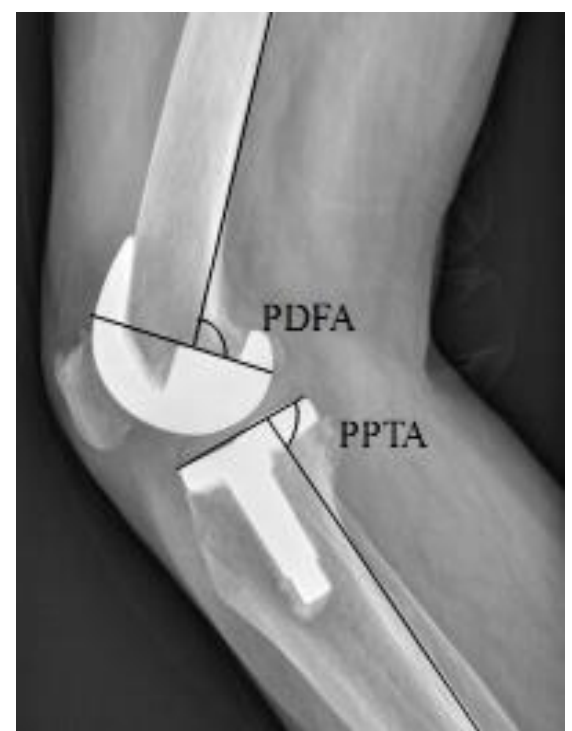

Fig2.2 Method for measuring PDFA and PPTA on the lateral radiographs 

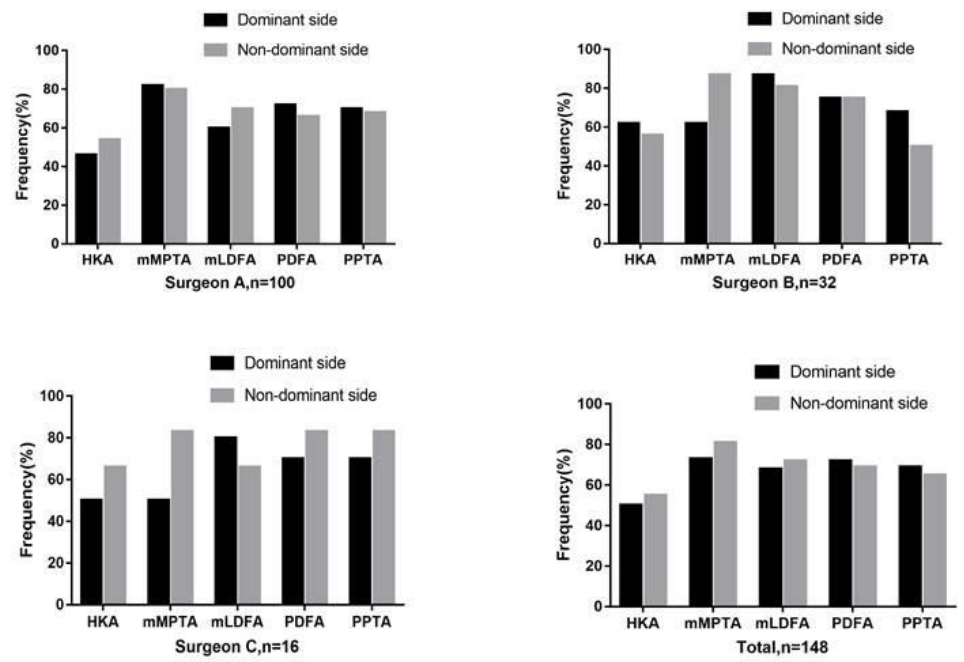

Fig.3 The percentage of safe zone placement for knee prostheses and both for dominant and non-dominant side TKA in surgeon A,B,C and all patients

Table 1 Characteristics of patients and knee prostheses

\begin{tabular}{lcccr}
\hline Item & $\begin{array}{c}\text { Dominant } \\
\text { side }\end{array}$ & $\begin{array}{c}\text { Non-dominant } \\
\text { side }\end{array}$ & t/ $\chi^{2}$ & P Value \\
\hline Age (years) & $68.3 \pm 6.5$ & $66.3 \pm 7.7$ & 1.743 & 0.083 \\
Gender (male/female) & $16 / 60$ & $11 / 61$ & 0.827 & 0.363 \\
BMI (kg/m ${ }^{2}$ ) & $26.4 \pm 3.3$ & $26.8 \pm 4.4$ & -0.710 & 0.479 \\
Preoperative HKA & $6.7 \pm 10.1$ & $9.4 \pm 8.7$ & -1.733 & 0.085 \\
Diagnosis, (n,\%) & - & - & 0.829 & 0.661 \\
Osteoarthritis & $73(49.3 \%)$ & $67(45.3 \%)$ & - & - \\
Rheumatoid arthritis & $2(1.4 \%)$ & $4(2.7 \%)$ & - & - \\
Traumatic arthritis & $1(0.7 \%)$ & $1(0.7)$ & - & - \\
Prosthesis type, (n, \%) & - & - & 2.329 & 0.676 \\
Zimmer Nex Gen LPS & $25(16.9 \%)$ & $22(14.9 \%)$ & - & - \\
LINK Gemini PS & $20(13.5 \%)$ & $23(15.5 \%)$ & - & - \\
Depuy PFC Sigma & $15(10.1 \%)$ & $14(9.5 \%)$ & - & - \\
AK-JPX(China) & $15(10.1 \%)$ & $10(6.8 \%)$ & - & - \\
GKPS(China) & $1(0.7 \%)$ & $3(2.0 \%)$ & - & - \\
\hline
\end{tabular}

Abbreviations: n number, BMI body mass index, HKA hip-knee-ankle angle

Table 2 The preoperative index in dominant and non-dominant side 


\begin{tabular}{llllllll}
\hline Groups & age $($ years $)$ & \multicolumn{2}{c}{ Gender } & & & \\
\cline { 3 - 6 } & & male & female & & & \\
\hline $\begin{array}{c}\text { Dominant } \\
(\mathrm{n}==76)\end{array}$ & $68.3 \pm 6.5$ & 16 & 60 & $26.4 \pm 3.3$ & $6.7 \pm 10.1$ & $85.2 \pm 4.1$ & $91.4 \pm 4.1$ \\
$\begin{array}{c}\text { Non-dominant } \\
(\mathrm{n}=72)\end{array}$ & $66.3 \pm 7.7$ & 11 & 61 & $26.8 \pm 4.4$ & $9.4 \pm 8.7$ & $84.8 \pm 5.2$ & $90.3 \pm 4.0$ \\
\hline$t$ & 1.743 & $\chi^{2}=0.827$ & $-0.710^{*}$ & -1.733 & 0.552 & 1.710 \\
$P$ & 0.083 & 0.363 & $0.479^{*}$ & 0.085 & 0.582 & 0.089 \\
\hline
\end{tabular}

Table 3 The post-operative data in dominant and non-dominant side

\begin{tabular}{lllllll}
\hline Groups & $\mathrm{n}(\mathrm{knee})$ & $\mathrm{HKA}$ & $\mathrm{mLDFA}$ & $\mathrm{mMPTA}$ & PDFA & PPTA \\
\hline Dominant & 76 & $2.1 \pm 4.2$ & $88.7 \pm 2.7$ & $89.5 \pm 2.7$ & $90.2 \pm 3.3$ & $84.8 \pm 2.8$ \\
Non-dominant & 72 & $1.6 \pm 4.1$ & $89.0 \pm 2.8$ & $89.9 \pm 2.7$ & $88.8 \pm 3.6$ & $85.4 \pm 3.7$ \\
\hline$t$ & - & 0.804 & -0.564 & -1.011 & 2.471 & -1.090 \\
$P$ & - & 0.423 & 0.574 & 0.314 & 0.015 & 0.278 \\
\hline
\end{tabular}


Figures

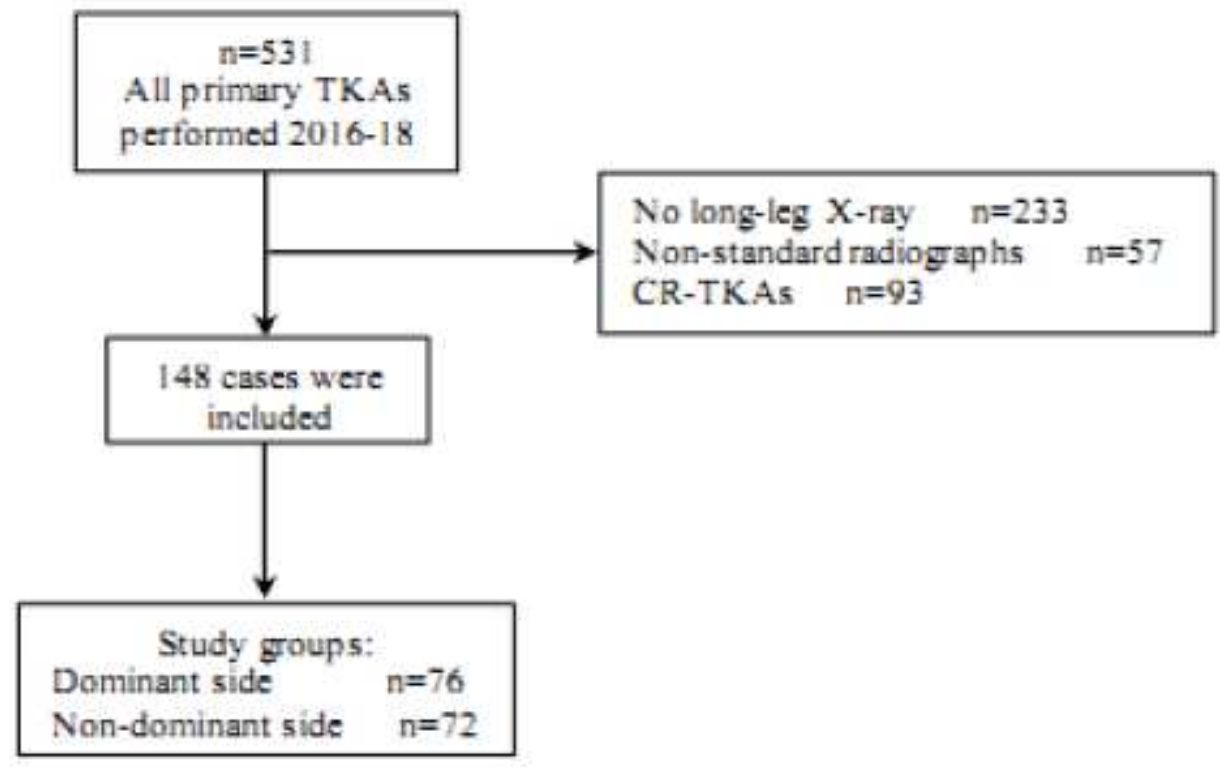

Figure 1

Study details TKA=total knee arthroplasty, CR-cruciate-retaining
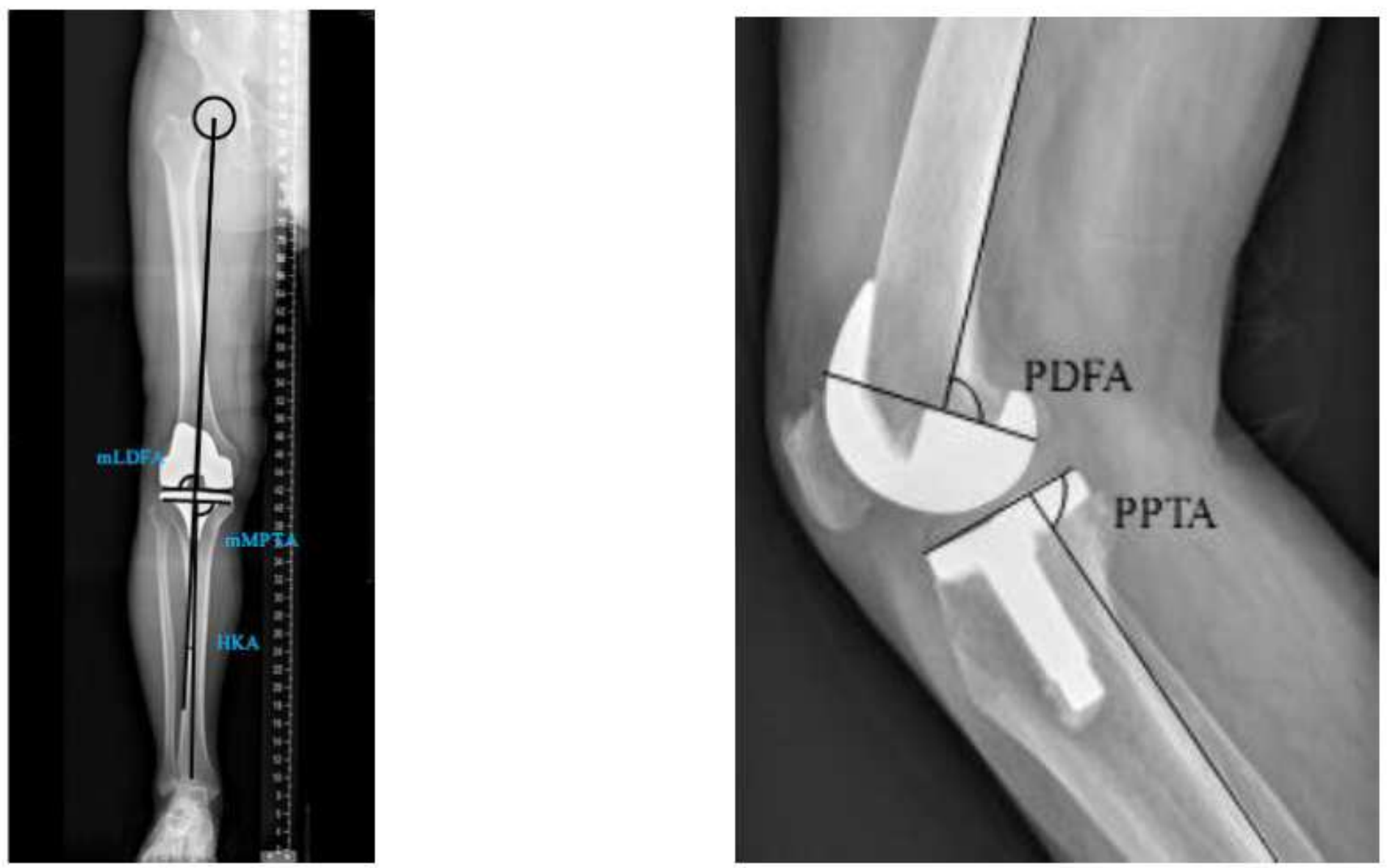
Figure 2

2.1 Method for measuring HKA,mLDFA and mMPTA on the full radiography. 2.2 Method for measuring PDFA and PPTA on the lateral radiographs
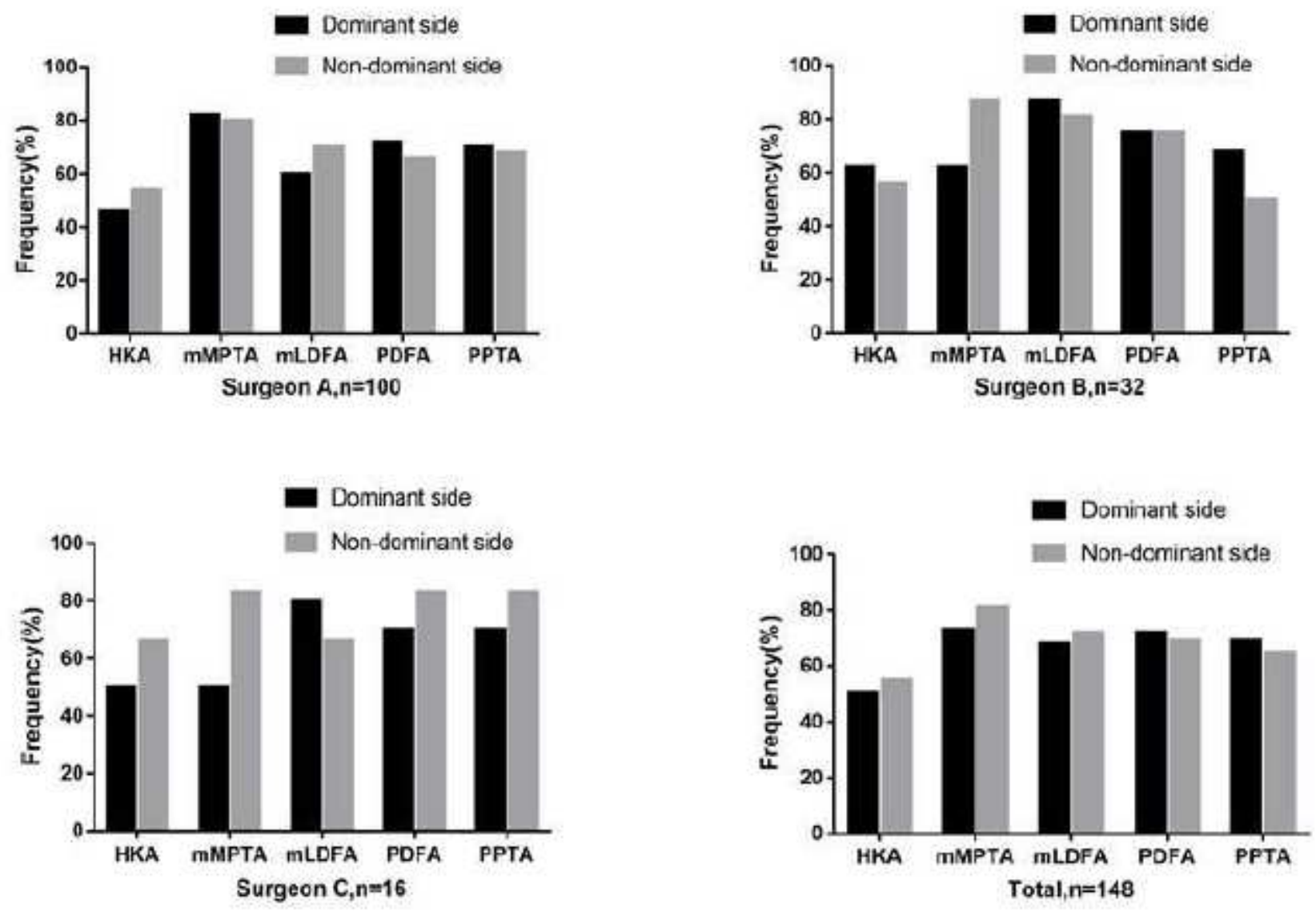

Figure 3

Fig.3 The percentage of safe zone placement for knee prostheses and both for dominant and non dominant side TKA in surgeon $A, B, C$ and all patients 